\title{
Using magnets for particle extraction from powder flow
}

\author{
Valerie Chopovda ${ }^{1} \quad$ Anton Gulley ${ }^{2}$ \\ Winston L Sweatman ${ }^{3}$
}

Received 16 November 2017; Revised 03 May 2018

\begin{abstract}
A model is explored for the extraction of ferromagnetic metallic particles using a magnetic filtration system. These metallic particles, which are transported by a powder flow may be fragments resulting from the standard wear and tear on industrial machinery. A project of this nature was brought to the 2015 Mathematics and Statistics in Industry NZ Study Group. Possible configurations of the magnetic filtration systems are considered with the target of better particle capture. The dependence of the model on the metallic particle's release zone and velocity is investigated. Monte-Carlo simulations are conducted for cases using perpendicular as well as parallel magnetic rods. The results confirm the efficacy of parallel offset rods and emphasise the importance of dense rod placement. The approach could be used to
\end{abstract}

DOI:10.21914/anziamj.v59i0.12642, (C) Austral. Mathematical Soc. 2018. Published August 24, 2018, as part of the Proceedings of the 13th Biennial Engineering Mathematics and Applications Conference. ISSN 1445-8810. (Print two pages per sheet of paper.) Copies of this article must not be made otherwise available on the internet; instead link directly to the DOI for this article. 
explore further alternative rod arrangements in advance of experimental implementation.

\section{Contents}

1 Introduction

C173

2 Mathematical model

C174

3 Simulations and results

C180

4 Concluding comments

C187

References

C188

\section{Introduction}

Small metallic particles arise in industrial food production through abrasion of metal components of the machinery [7]. These particles may be magnetised in the process. The use of magnetic filtration is just part of a broad suite of contaminant identification and removal methods that ensure that food products meet strict quality controls. In the magnetic filtration process powder flows down a vertical chute containing rows of horizontal, evenlyspaced magnetic rods perpendicular to the flow. Metallic particles are captured on the rods from which they are removed.

The process of magnetic separation is used in a number of industries, from mineral processing to food processing. Sometimes the separated metallic particles are the desired products but not necessarily. Magnetic separators differ according to the type of product and the manufacturing process. In previous research, Kopp considered a type of roll magnetic separator for the case of viscous flow [6]. He also discussed the influence of collisions between 
magnetic particles. Svoboda and Fujita gave an overview of the underlying physics and review different magnetic separators, with a particular emphasis on wet magnetic separators that have been used in the mineral processing industry [10]. Stradling considered dry magnetic separators and described the physics behind the dry roll magnetic separator [9]. A mathematical model for a crossbelt and induced magnetic roll separators was introduced. Noguchi and Kim considered a mathematical model that takes into account the magnetic field, magnetic fluid flow and diffusion of magnetic particles into the flow [8]. Their model is general rather than for a specific type of magnetic separator.

The present investigation originally arose from a 2015 Mathematics and Statistics in Industry NZ Study Group (MINZ 2015) project brought by the Fonterra group [3]. Building upon this earlier work, we explore the efficacy of alternative arrangements of magnetic rods for removing metallic particles from the milk powder flow. The results confirm the effectiveness of the present arrangement with parallel offset magnetic rods. They also show the importance of a dense arrangement of rods for generating a strong magnetic field and trapping the metallic particles.

\section{Mathematical model}

We use the mathematical model developed for the system considered at MINZ 2015 [3] with small modifications and enhancements. This model is constructed from a number of components. Parameters and variables are listed in Table 1.

Magnetic filtration system A sketch of the magnetic filtration system is presented in Figure 1. The filtration system is enclosed in a prism of square cross-section of side $32 \mathrm{~cm}$. Milk powder flows down into the filtration system through a circular pipe of radius of $15 \mathrm{~cm}$. We focus on the flow down the main body of the magnetic filtration system and do not consider any effects 
Table 1: Variables and parameters used in the model

\begin{tabular}{|l|l|l|l|}
\hline Symbol & Quantity & Unit & Value \\
\hline $\mathrm{n}$ & number of dipoles in a rod & - & 7 \\
\hline $\mathrm{L}$ & length of a dipole & $\mathrm{m}$ & 0.023 \\
\hline $\mathrm{a}$ & radius of a rod & $\mathrm{m}$ & 0.0125 \\
\hline $\mathrm{s}$ & separation between sides of two rods & $\mathrm{m}$ & 0.056 \\
\hline $\mathrm{r}_{\text {pipe }}$ & radius of hopper & $\mathrm{m}$ & 0.15 \\
\hline$\mu_{0}$ & magnetic permeability constant & $\mathrm{N} \mathrm{m}^{-1}$ & $4 \pi \times 10^{-7}$ \\
\hline $\mathrm{M}$ & dipole moment & $\mathrm{N} \mathrm{m} \mathrm{T}^{-1}$ & $0.0055 \times 10^{7}$ \\
\hline$\chi_{\mathrm{a}}$ & volume fraction of air & - & 0.9 \\
\hline$\chi_{\mathrm{m} p}$ & volume fraction of milk powder & - & 0.1 \\
\hline $\mathrm{r}_{\mathrm{m} \mathfrak{p}}$ & milk powder particle radius & $\mathrm{mm}$ & $1 \times 10^{-8}$ \\
\hline$\rho_{\mathrm{m} p}$ & milk powder bulk density & $\mathrm{kg} \mathrm{m}^{-3}$ & 1150 \\
\hline$\rho_{\mathrm{a}}$ & density of air & $\mathrm{kg} \mathrm{m}^{-3}$ & 1.225 \\
\hline$\rho_{\mathrm{f}}$ & total density of the flow & $\mathrm{kg} \mathrm{m}^{-3}$ & 116 \\
\hline$\eta_{\mathrm{a}}$ & dynamic viscosity of air & $\mathrm{kg} \mathrm{m}^{-1} \mathrm{~s}^{-1}$ & $1.846 \times 10^{-5}$ \\
\hline$\eta_{\mathrm{f}}$ & dynamic viscosity of mixture & $\mathrm{kg} \mathrm{m}^{-1} \mathrm{~s}^{-1}$ & $2.307 \times 10^{-5}$ \\
\hline$\nu_{\mathrm{f}}$ & flow velocity & $\mathrm{m} \mathrm{s}^{-1}$ & 1 \\
\hline $\mathrm{d}$ & diameter of a particle & $\mathrm{mm}$ & 1 \\
\hline$\rho_{\mathrm{p}}$ & metal particle density & $\mathrm{kg} \mathrm{m}^{-3}$ & 8000 \\
\hline$\nu_{\mathrm{p}}$ & initial particle velocity & $\mathrm{m} \mathrm{s}^{-1}$ & $\mathrm{same} \mathrm{as} v_{\mathrm{f}}$ \\
\hline$\Delta \chi$ & particle relative magnetic susceptibility & - & 40 \\
\hline
\end{tabular}

due to the enclosing walls. The magnetic rods are constructed from several bar magnet dipoles placed end to end with like poles adjacent, e.g. in our model we have a north (N) and south (S) arrangement NS-SN-NS-SN-NS-SN-NS (Figure 2). The rods are arranged in two horizontal layers. Within each layer the rods are parallel and equally spaced.

Initially, four preliminary arrangements of rods were investigated. After obtaining the results from these, and investigating minor variations, four further, similar rod arrangements were trialled.

The first two arrangements considered resemble that chosen at MINZ 2015, following discussions with the Fonterra group industry representative, and have a similar structure to those currently used [3]. The rods in different 


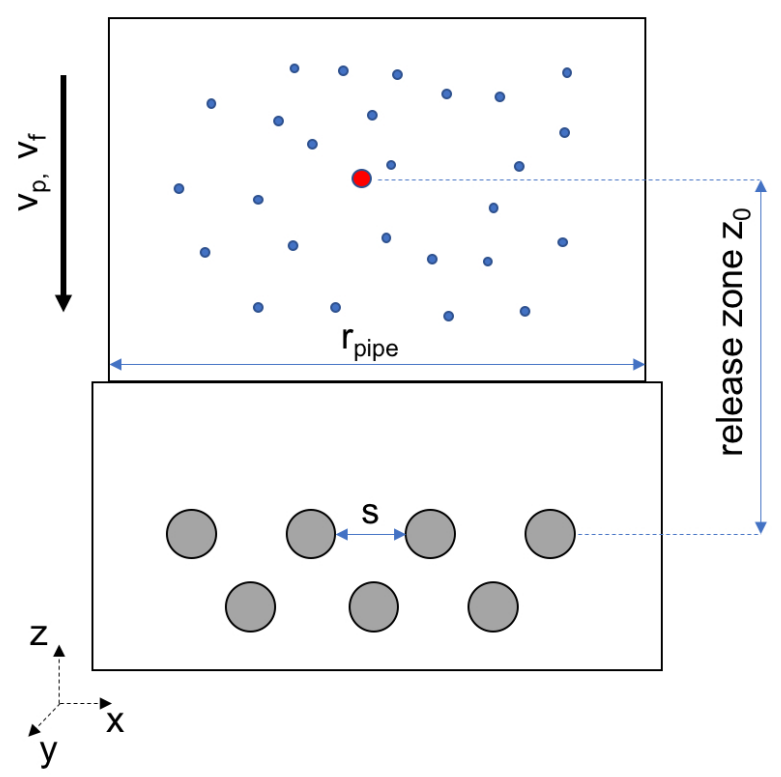

Figure 1: Side view of the magnetic filtration system perpendicular to the magnetic rods. The rod arrangement (b) is shown here. The magnetic rods are shown as grey circles. Blue dots indicate milk powder particles and the red dot is a metallic particle trapped in the milk powder flow.

layers are parallel but offset, so that a rod in the top layer is above a space in the bottom layer. In arrangement (a) there are 3 rods on the top and 4 rods on the bottom. In arrangement (b) there are 4 rods on the top and 3 rods on the bottom layer. In both arrangements (a) and (b), the spacing between the layers was chosen so that the distance between rods in different layers was the same as that between the rods within the same layer. In the third (c) and fourth (d) arrangements, the separation of the layers is the same but the direction of the rods in the two layers is perpendicular, forming a sieve-like structure. The third arrangement (c) has 3 rods on the top and 3 rods on the bottom while the fourth (d) has 4 rods on the top and 4 rods on the bottom. 


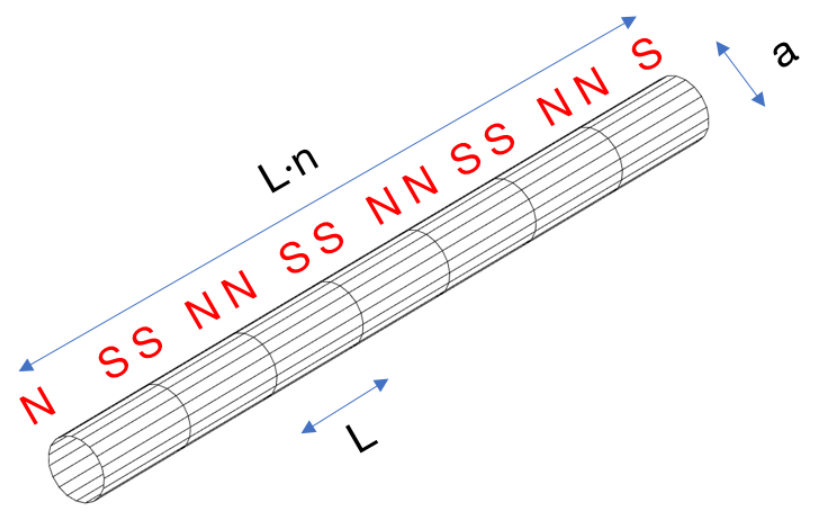

Figure 2: The structure of a magnetic rod.

Powder flow By volume, the powder flow is $90 \%$ air and $10 \%$ milk powder. Any metallic particles present have a negligible percentage and are assumed not to affect the flow. The mixture density is $\rho_{f}=x_{a} \rho_{a}+x_{m p} \rho_{m p}$. Dynamic viscosity of the mixture is calculated using the Einstein relation [4], [5, pp. 3662 ], for the viscosity of a liquid containing a suspension of insoluble tiny rigid bodies,

$$
\eta_{\mathrm{f}}=\eta_{\mathrm{a}}\left(1+2.5 x_{\mathrm{mp}}\right)=1.25 \eta_{\mathrm{a}}
$$

Flow rates differ between milk powder production facilities. They are roughly of the order 2.5-25 tonnes per hour [3]. Using the physical dimensions modelled here, the volumetric flow rate $\mathrm{Q}_{v}=0.0059-0.059 \mathrm{~m}^{3} \mathrm{~s}^{-1}$. The flow enters the magnetic filtration system through a circular pipe. From the cross-sectional area $A=\pi r_{\text {pipe }}^{2}$, the velocity of the incoming powder mixture $v_{\mathrm{f}}=\mathrm{A} / \mathrm{Q}_{v}$ is estimated to be in the range $0.084-0.84 \mathrm{~m} \mathrm{~s}^{-1}$. The Reynolds number is in the range $5278-52784$. The flow leaves the confined pipe to pass into the box containing the magnetic rods. With the high estimates for Reynolds number, the flow is treated as inviscid incompressible irrotational steady flow. For the simulations, the flow rate in the model is set in the upper range of expected velocity. The flow is modelled as uniform potential flow 
around magnetic cylinders with no-slip boundary conditions.

In reality there will be a boundary layer around the rods, however, for this model, we assume that this does not influence the magnetic capture. Model simulations indicate that most metallic particles are affected, and subsequently captured, when they are above or at a rod's level, where any boundary-layer effect will be minimal.

Magnetic field The rods are constructed from multiple magnetic dipoles. The components of the magnetic field around a single dipole $\mathbf{B}$ [1] are

$$
\begin{aligned}
& B_{y}=-\frac{\mu_{0} M}{4 \pi} \int_{0}^{2 \pi} \int_{0}^{a}\left(\frac{R(L / 2-y)}{\beta^{(-)}}+\frac{R(L / 2+y)}{\beta^{(+)}}\right) d R d \Phi \\
& B_{\rho}=-\frac{\mu_{0} M}{4 \pi} \int_{0}^{2 \pi} \int_{0}^{a}\left(-\frac{R(2 \rho-2 R \cos \Phi)}{2 \beta^{(-)}}+\frac{R(2 \rho-2 R \cos \Phi)}{2 \beta^{(+)}}\right) d R d \Phi
\end{aligned}
$$

in cylindrical coordinates $(\rho, \theta, y)$, with $\rho=\sqrt{x^{2}+z^{2}}$, where

$$
\begin{aligned}
& \beta^{(+)}=\left(R^{2}+(L / 2-y)^{2}+\rho^{2}-2 R \rho \cos \Phi\right)^{3 / 2}, \\
& \beta^{(-)}=\left(R^{2}+(L / 2+y)^{2}+\rho^{2}-2 R \rho \cos \Phi\right)^{3 / 2} .
\end{aligned}
$$

Metallic particles The metallic particles are assumed to be from stainless steel food processing equipment and to be spherical of diameter $1 \mathrm{~mm}$. This size was chosen in discussion with Fonterra representatives at the mathematicsin-industry study group (MINZ2015): large particles are more likely to avoid being trapped by magnetic filtration because of their higher momentum, however, particles larger than $1 \mathrm{~mm}$ are removed using other methods. The density of the particles is taken to be $\rho_{\mathrm{p}}=8000 \mathrm{~kg} \mathrm{~m}^{-3}$ [3]. Although the metal is initially non-magnetic, the particles entering the powder flow are magnetised by strain-induced martensitic transformation [7]. In the model we set relative magnetic susceptibility $\Delta \chi=40$. Metallic particles are assumed to enter the circular pipe leading to the magnetic filtration system with the same velocity as the powder flow. 
Three forces influence the motion of a metallic particle: the magnetic force due to the rods, gravity, and drag [3, 7],

$$
\mathbf{F}_{\text {total }}=\mathbf{F}_{\mathrm{m}}+\mathbf{F}_{\mathrm{g}}+\mathbf{F}_{\mathrm{d}},
$$

where

$$
\begin{aligned}
\mathbf{F}_{\mathrm{m}} & =\frac{\Delta \chi \mathrm{V}_{\mathrm{p}}}{\mu_{0}}(\mathbf{B} \cdot \nabla) \mathbf{B}, \\
\mathbf{F}_{\mathrm{g}} & =\left(\rho_{\mathrm{p}}-\rho_{\mathrm{f}}\right) \mathrm{V}_{\mathrm{p}} \mathbf{g}, \\
\mathbf{F}_{\mathrm{d}} & =6 \pi \eta_{\mathrm{f}} \mathrm{r}\left(\mathbf{v}_{\mathbf{f}}-\mathbf{v}_{\mathrm{p}}\right),
\end{aligned}
$$

with particle volume $V_{p}$ and gravitational acceleration $\mathbf{g}$.

From the equations (3) the components of the metallic particle's acceleration are derived:

$$
\begin{aligned}
\mathrm{a}_{\mathrm{m}} & =\frac{\Delta x}{\rho_{\mathrm{p}} \mu_{0}}(\mathbf{B} \cdot \nabla) \mathbf{B} \\
\mathrm{a}_{\mathrm{g}} & =-\mathbf{g}, \\
\mathrm{a}_{\mathrm{d}} & =\frac{6 \pi \eta_{\mathrm{f}} \mathrm{r}}{\rho_{\mathrm{p}} \mathrm{V}_{\mathrm{p}}}\left(\mathbf{v}_{\mathrm{f}}-\mathbf{v}_{\mathrm{p}}\right),
\end{aligned}
$$

where $\rho_{\mathrm{f}}$ is treated as negligible in comparison to $\rho_{\mathrm{p}}$.

Milk powder is tiny compared to the metallic particles, and the powder flow is mostly air, so it is unlikely that the powder has much effect on the metallic particles. It would be different with smaller metallic particles. Following the previous magnitude of forces computations [3], $\mathrm{a}_{\mathrm{d}}\left(\sim 10^{-3} \mathrm{~m} \mathrm{~s}^{-2}\right)$ is considerably smaller than $\mathrm{a}_{\mathrm{m}}\left(\sim 10^{0} \mathrm{~m} \mathrm{~s}^{-2}\right)$ and $\mathrm{a}_{\mathrm{g}}\left(\sim 10^{1} \mathrm{~m} \mathrm{~s}^{-2}\right)$. As the drag force is much less significant than the other forces, it is sufficient to approximate the flow as being homogenous with velocity $1 \mathrm{~m} \mathrm{~s}^{-1}$ downwards for computing the drag.

Figures 3, 4, 5, 6 and 7 show the magnetic acceleration of a metallic particle for various arrangements of rods. Arrangements (a) and (b) lead to magnetic fields that are symmetric in both $\boldsymbol{x}$ - and $\boldsymbol{y}$-directions. However, in arrangements (c) and (d) there is a loss of symmetry due to the different interactions of similar 


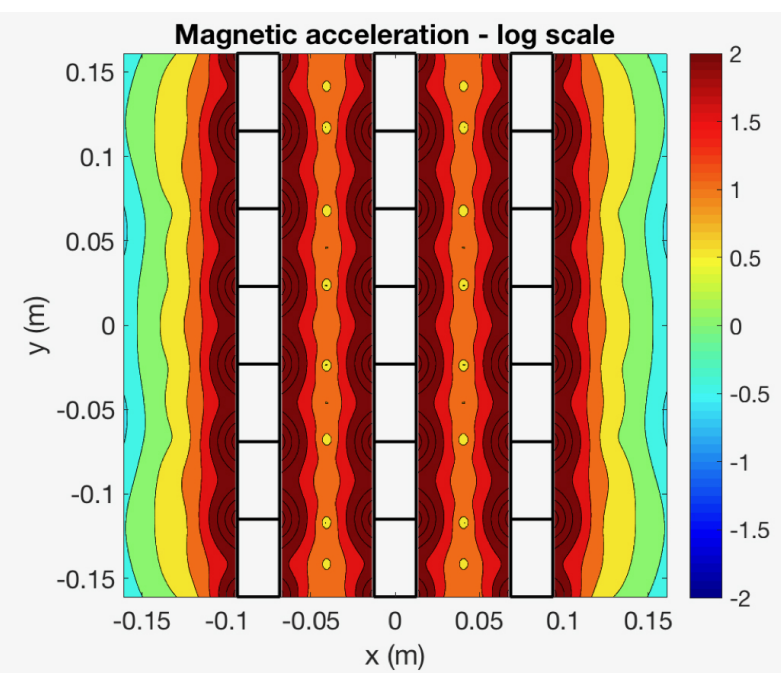

Figure 3: Magnetic acceleration for a metallic particle $-x-y$ cross-section through the top layer for rod arrangement (a). White boxes indicate the magnetic dipoles that make up the magnetic bars.

and opposite poles. If the rods had contained an even number of dipoles then these sieve-like arrangements would also have the $x$ - and $y$-directional symmetry. In arrangements (c) and (d), it is striking how similar the fields are between the rods where the magnetic field is dominated by the closest rods.

\section{$3 \quad$ Simulations and results}

Particle paths through the magnetic array are simulated by numerical integration using the improved Euler's method [2, pp. 86-87]. For each of the arrangements of rods, Monte-Carlo simulations are performed for three different particle release heights: 6,10 and $40 \mathrm{~cm}$ above the first layer of rods. These represent different lengths of circular pipe above the magnetic filtration 

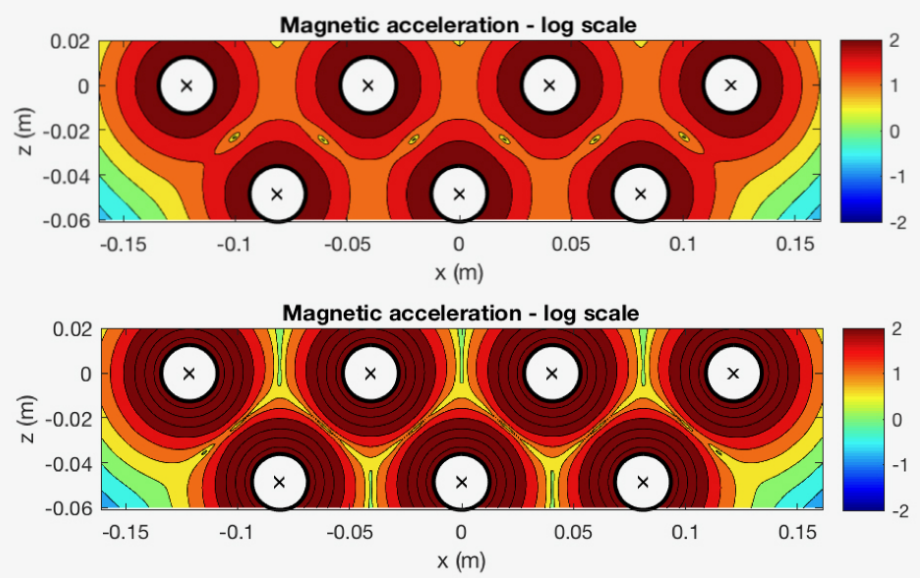

Figure 4: Magnetic acceleration for a metallic particle $-x-z$ cross-sections for rod arrangement (b). (Top) cross-section taken midway through a dipole, (Bottom) cross-section taken at the magnetic poles between dipoles.

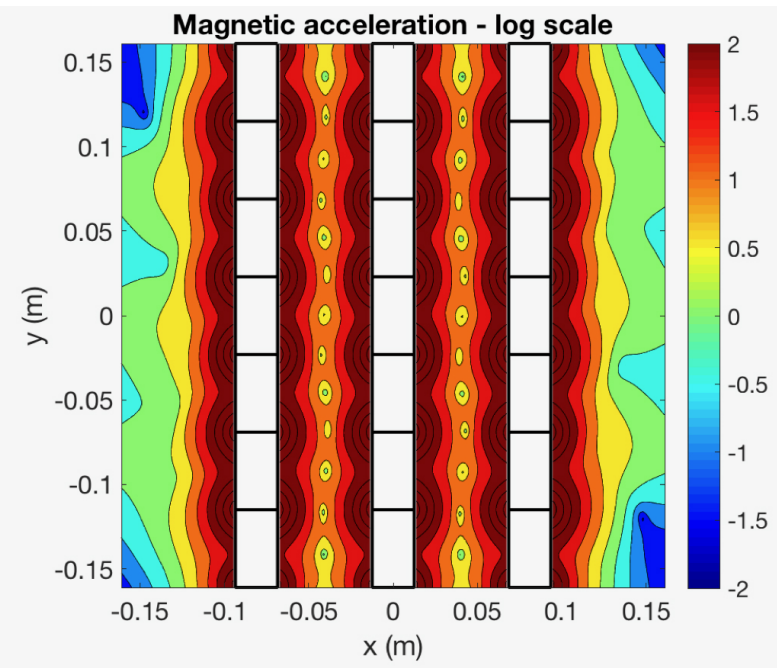

Figure 5: Magnetic acceleration for a metallic particle - $x-y$ cross-section through the top layer for the sieve-like rod arrangement (c). 

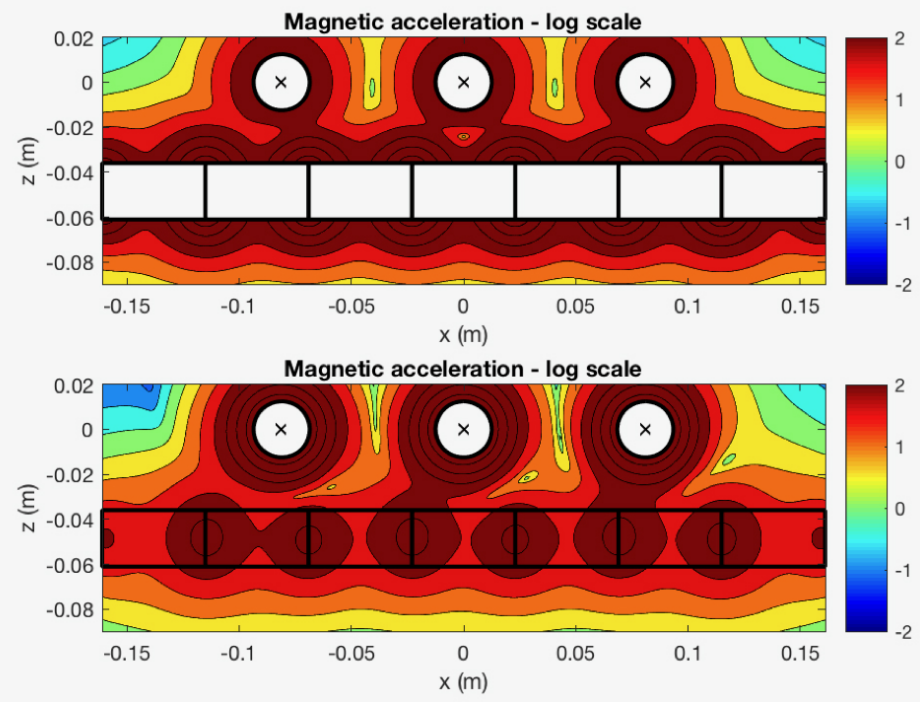

Figure 6: Magnetic acceleration for a metallic particle $-x-z$ cross-sections for rod arrangement (c). (Top) cross-section taken midway through a dipole in the top layer and through a rod in the lower layer, (Bottom) cross-section taken at the magnetic poles between dipoles in the top layer and between rods in the lower layer.

system. There were approximately 1000 simulations for each case performed over a grid on a quadrant (usually the first quadrant $0<x<15 \mathrm{~cm}$ and $0<y<15 \mathrm{~cm}$ ). Table 2 shows the particle capture rates. The standard error is $0.5 \%$.

For all rod arrangements, the results consistently show that higher release zones lead to lower metallic particle capture. The higher the initial position of the particle, the higher its speed when it reaches the rod layers. This increased speed reduces the opportunity for capture [3].

There is only a marginal difference between the two parallel arrangements (a) and (b). The results suggest that it may be slightly more effective to have 


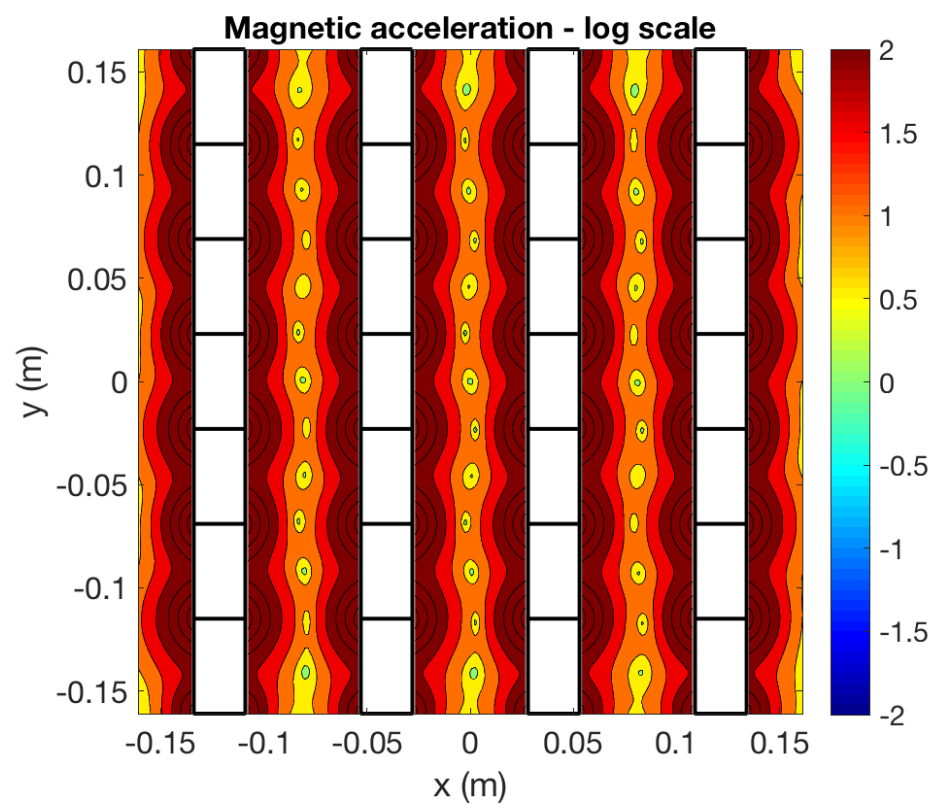

Figure 7: Magnetic acceleration for a metallic particle - $x-y$ cross-section through the top layer for the sieve-like rod arrangement (d).

more rods in the lower layer. Individual particle trajectories in Figure 8 show that the upper layer of rods may produce some sideways spreading of the particles in the $x$-direction which may provide some explanation. The spread particles cannot be trapped if there are no rods nearby.

Other particle trajectories for the perpendicular arrangement (c) are shown in Figure 9. Trajectories where the particle is not captured tend to go through gaps where the magnetic field is weakest perhaps caused by destructive magnetic interference [3].

Due to the loss symmetry in the sieve-like configurations (c) and (d), as illustrated by Figures 5 to 7 , extra simulations were performed on the second quadrant $-15<x<0 \mathrm{~cm}$ and $0<y<15 \mathrm{~cm}$ for these arrangements. 
Table 2: Capture rates for different release zones and rod arrangements

\begin{tabular}{|l|l|l|l|l|l|}
\hline & $\begin{array}{l}\text { Rod } \\
\text { arrangement }\end{array}$ & Quadrant & $\begin{array}{l}\text { Release zone } \\
z_{0}=6 \mathrm{~cm}\end{array}$ & $\begin{array}{l}\text { Release zone } \\
z_{0}=10 \mathrm{~cm}\end{array}$ & $\begin{array}{l}\text { Release zone } \\
z_{0}=40 \mathrm{~cm}\end{array}$ \\
\hline (a) & $\begin{array}{l}\text { parallel } \\
3 \text { over 4 }\end{array}$ & 1 st & $92.8 \%$ & $91.3 \%$ & $81.3 \%$ \\
\hline (b) & $\begin{array}{l}\text { parallel } \\
4 \text { over 3 }\end{array}$ & 1 st & $92.5 \%$ & $90.3 \%$ & $80.2 \%$ \\
\hline (c) & $\begin{array}{l}\text { perpendicular } \\
3 \text { over 3 }\end{array}$ & $\begin{array}{l}\text { 1st } \\
\text { 2nd }\end{array}$ & $\begin{array}{l}82.3 \% \\
83.2 \%\end{array}$ & $\begin{array}{l}79.2 \% \\
80.4 \%\end{array}$ & $\begin{array}{l}67.9 \% \\
67.9 \%\end{array}$ \\
\hline (d) & $\begin{array}{l}\text { perpendicular } \\
\text { over 4 }\end{array}$ & $\begin{array}{l}\text { 1st } \\
\text { 2nd }\end{array}$ & $\begin{array}{l}95.5 \% \\
94.1 \%\end{array}$ & $\begin{array}{l}93.5 \% \\
92.2 \%\end{array}$ & $\begin{array}{l}82.7 \% \\
82.0 \%\end{array}$ \\
\hline (e) & $\begin{array}{l}\text { parallel } \\
3 \text { over 3 }\end{array}$ & 1 st & $75.0 \%$ & $72.1 \%$ & $61.5 \%$ \\
\hline (f) & $\begin{array}{l}\text { parallel } \\
4 \text { over 4 }\end{array}$ & 1 st & $89.7 \%$ & $86.7 \%$ & $78.1 \%$ \\
\hline (g) & $\begin{array}{l}\text { perpendicular } \\
3 \text { over 4 }\end{array}$ & 1st & $89.5 \%$ & $87.5 \%$ & $74.4 \%$ \\
\hline (h) & $\begin{array}{l}\text { perpendicular } \\
4 \text { over 3 }\end{array}$ & 1 st & $90.4 \%$ & $88.1 \%$ & $75.8 \%$ \\
\hline
\end{tabular}

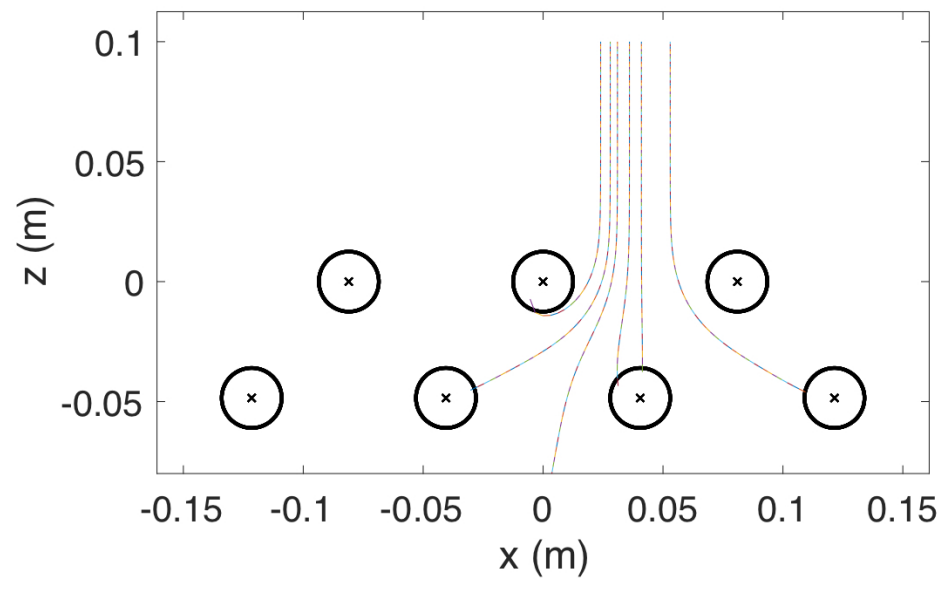

Figure 8: Individual particle paths $10 \mathrm{~cm}$ release, arrangement (a). 

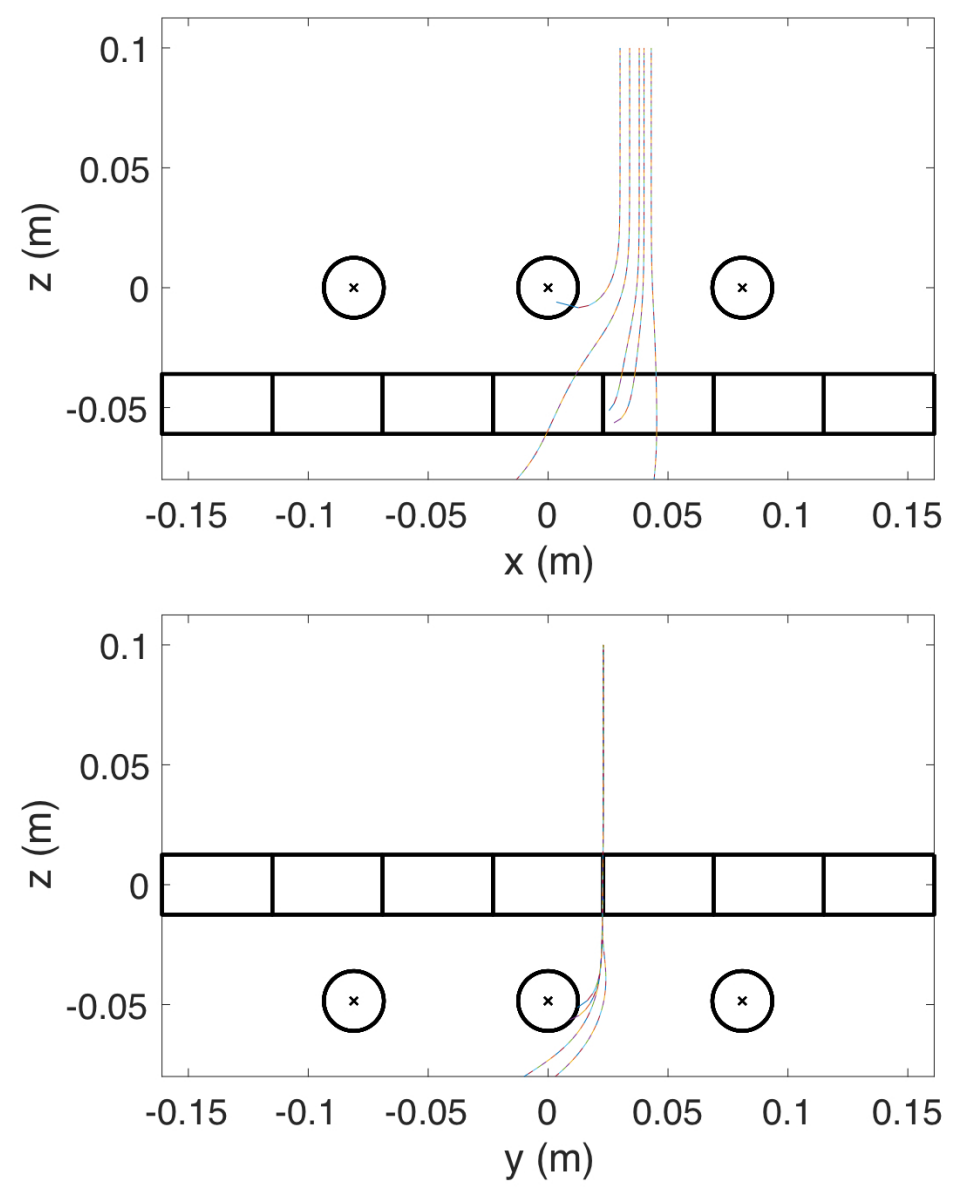

Figure 9: Individual particle paths $10 \mathrm{~cm}$ release, arrangement (c). The $x-z$ and $y-z$ projections are shown. 
These are presented separately in Table 2 for comparison. There is a modest difference between the measured first and second quadrant values of at most $1.5 \%$. This difference becomes insignificant as the release height increases to $40 \mathrm{~cm}$.

The sieve-like rod arrangement (c) with three rods in both layers shows the worst capture rate of all the preliminary arrangements ((a)-(d)). In contrast, the sieve-like rod arrangement (d) with an extra rod in each layer had the best capture rate. This suggests that the difference in capture rates in these preliminary arrangements is more due to the density or equivalently number of magnetic rods present (eight for (d), seven each for (a) and (b) and six for (c)) than due to whether the rods are parallel or perpendicular. To further explore these effects, arrangements (e)-(h), included in Table 2, were investigated.

Arrangements (e) and (f) are similar to arrangements (a) and (b) in that the rods are all parallel but differ in that the rods are not offset. In comparison with arrangements (a) and (b) we see some advantage in having more rods but this effect is less significant than the effect of the lack of offset. Despite an extra rod, arrangement (f) has a worse capture rate than arrangements (a) and (b). Arrangement (e) with both one less rod and the lack of offset is much worse than (a) and (b).

Arrangements (g) and (h) are variants on the perpendicular arrangements (c) and (d), however, they have only seven rods like arrangements (a) and (b). The poorer performance by both of arrangements (g) and (h) than either of arrangements (a) and (b) indicates that the parallel offset arrangement is better than a perpendicular arrangement providing that there is a similar density of rods.

As well as arrangements (a)-(h), we performed a single test on a variant of arrangement (a) with twice the gap size between layers. The capture rate for that simulation decreased significantly to $63.7 \%$ for the release height of 6 $\mathrm{cm}$. This is also substantially lower than the capture rate for arrangement (a) with a release height of $40 \mathrm{~cm}$ even though all the magnetic rods are lower than either of the double-gap layers. This indicates that in this specific case 
it is not the speed of the metallic particle that is the dominant factor in its capture. The increased gap weakens the magnetic field between the two layers so that this is less able to divert the metallic particle to its capture on a rod. It is important that the layers of rods are situated close to one another.

However, another potential influence is the positioning of the gaps in the lower layer. Particles that are not captured by the upper layer tend to be diverted sideways. If the gaps in the lower layer occur in the path of these particles then they will not be captured. It may be that the exact positioning of the rods and gaps in the lower layer of the double-spaced simulation is having an effect.

\section{Concluding comments}

We have considered the use of magnetic rods to recover metallic particles transported within a milk powder flow. We explored various arrays of layers of magnetic rods using a simple mathematical model and numerical simulation. The simulations indicate that, out of the arrangements explored, the current arrangement of parallel offset magnetic rods is the most effective. A higher density of rods improves the capture rate both because there are more rods to trap the particles upon and because the closer rods create a stronger magnetic field. Experimental data and validation is needed to confirm these results.

The filtration system will work most effectively if the rods in the lower layer are placed on the main path of particles that are not captured by the upper layer. If particles are falling directly down then this favours the parallel offset arrangements such as (a) and (b). However, as noted in Section 3, the upper layer of rods may also produce sideways deviation of the particles as illustrated by Figure 8. This deviation is also dependent upon the placement of the rods in the lower layer as they affect the magnetic field. While it seems necessary to have the layers of rods close together, it may be possible to optimise the exact vertical gap between the layers. Before embarking on this 
process it would be desirable to have more precise experimental data.

\section{References}

[1] S.M. Blinder (2011) Magnetic Field of a Cylindrical Bar Magnet Wolfram Demonstrations Project. http://demonstrations. wolfram. com/MagneticFieldOfACylindricalBarMagnet/ C178

[2] J.C. Butcher (2008) Numerical Methods for Ordinary Differential Equations, John Wiley \& Sons Ltd., England. C180

[3] H. Cooper, J. Denier, A. Ali, V. Chopovda, A. Gulley (2017) Modelling the effectiveness of magnets in particle extraction, ANZIAM Journal (2017), 57: M332-M352. doi:10.21914/anziamj.v57i0.12037 C174, C175, C177, C178, C179, C182, C183

[4] A. Einstein (1906) Eine neue Bestimmung der Moleküldimensionen, Ann. Phys., 324: 289-306. C177

[5] A. Einstein, R. Fürth (1893-1956) Investigations on the theory of the Brownian movement, Dover. C177

[6] J. Kopp (1983) The physics of falling curtain dry magnetic separation, International Journal of Mineral Processing, 10, 297-308. doi:10.1016/0301-7516(83)90019-4 C173

[7] Y. Nakai, F. Mishima, Y. Akiyama, S. Nishijima (2010) Development of Magnetic Separation System for Powder Separation, IEEE Transactions on applied superconductivity, 20:3, 941-944. doi:10.1109/TASC.2010.2043086 C173, C178, C179

[8] S. Noguchi, S. Kim (2011) Development of a numerical simulation method for the magnetic separation of magnetic particles, IEEE Transactions on magnetics, 47:5, 898-901. doi:10.1109/CEFC.2010.5481745 C174 
[9] A.W. Strandling (1993) The physics of open-gradient dry magnetic separation, International Journal of Mineral Processing, 39, 1-18. doi:10.1016/0301-7516(93)90048-F C174

[10] J. Svoboda, T. Fujita (2003) Recent developments in magnetic methods of material separation, Minerals Engineering, 16, 785-792. doi:10.1016/S0892-6875(03)00212-7 C174

\section{Author addresses}

1. Valerie Chopovda, Institute of Natural and Mathematical Sciences, Massey University, Auckland, New Zealand.

mailto:valerie.chopovda@gmail.com

2. Anton Gulley, The University of Auckland, New Zealand. mailto:gullant@gmail.com

3. Winston L Sweatman, Institute of Natural and Mathematical Sciences, Massey University, Auckland, New Zealand. mailto:w.sweatman@massey.ac.nz 